\title{
On the Dynamical detection of Majorana fermions in current-biased nanowires
}

\author{
Fernando Domínguez, ${ }^{1}$ Fabian Hassler, ${ }^{2}$ and Gloria Platero ${ }^{1}$ \\ ${ }^{1}$ Instituto de Ciencia de Materiales, CSIC, Cantoblanco, E-28049 Madrid, Spain \\ ${ }^{2}$ Institute for Quantum Information, RWTH Aachen University, 52056 Aachen, Germany
}

(Dated: August 2, 2018)

\begin{abstract}
We analyze the current-biased Shapiro experiment in a Josephson junction formed by two onedimensional nanowires featuring Majorana fermions. Ideally, these junctions are predicted to have an unconventional $4 \pi$-periodic Josephson effect and thus only Shapiro steps at even multiples of the driving frequency. Taking additionally into account overlap between the Majorana fermions, due to the finite length of the wire, renders the Josephson junction conventional for any dc-experiments. We show that probing the current-phase relation in a current biased setup dynamically decouples the Majorana fermions. We find that besides the even integer Shapiro steps there are additional steps at odd and fractional values. However, different from the voltage biased case, the even steps dominate for a wide range of parameters even in the case of multiple modes thus giving a clear experimental signature of the presence of Majorana fermions.

PACS numbers: 73.23.-b, 05.60.Gg
\end{abstract}

Majorana Fermions (MFs) have recently been predicted to occur in a multitude of different condensedmatter systems [1 [5, 5, 6, 8]. The interest in MFs stems from the non-Abelian quantum statistics which forms the basis of topological quantum computation [2, 4, 9, 10]. Majorana fermions naturally occur in half-vortices of chiral $p$-wave superconductors. Although this type of superconductivity has not been found, it was realized recently that $s$-wave superconductor together with strong spin-orbit and an applied magnetic field may emulate a $p$ wave superconductor $5,5,6,611$. During the last months three different experiments 12 14] appeared in the literature which may provide the first experimental evidence of MFs.

Signatures of Majorana Fermions appear in the electrical [15 17] and thermal conductance [18, 19], shot-noise [19], Andreev-reflection [16, 20] and the non-local tunneling [16, 21 23]. In this Letter we will focus on the measurement of the fractional Josephson effect, given when we put together two superconductors featuring MFs [1, 4 6, 26 30]. Physically, this effect is produced by the fact that in the presence of a Majorana bound mode, the supercurrent carries single electrons instead of the usual Cooper pairs. Thus, this fractional Cooper pairs affect the supercurrent by turning it from $\sin (\varphi)$ to $\sin (\varphi / 2)$.

In Josephson junctions, Shapiro step experiments allow for the deduction of the periodicity of the current-phase relation of the junction [31, 32]. Very recently, Shapirosteps have been analyzed for voltage-biased Majorana wires 33 35]. However, the more experimentally realistic current-biased experiment [31] remains unexplored.

In one-dimensional (1D) Majorana wire, MFs will appear at the end points [1]. In an ideal situation, the ends are infinitely apart from each other avoiding their recombination. In turn, when the wire is finite, the overlap, although very small, is different from zero, thus MF pair recombines and the special properties that the MF confer to the system are lost immediately [36]. Physically, one can circumvent this problem using a Josephson junction where the gauge invariant phase is tuned non-adiabatically. In this way, transitions between the recombined fermions induce a dynamical decoupling into Majorana fermions.

In this work we analyze theoretically the current biased Shapiro experiment 32] in a finite 1D Josephson junction where the MFs are recombined (see Fig. (1). In the presented setup, the current bias the gauge invariant phase, inducing dynamical decoupling of the MFs. Meanwhile, it induces a voltage difference that can be measured, presenting the pattern of the periodicity of the junction. We have calculated the induced voltage by means of the Resistively Shunted Junction (RSJ) model [31]. In addition, we include extra Andreev modes carrying a $2 \pi$ periodic current and quasiparticle poisoning (QP).

In contrast to the infinite length case, where only even Shapiro steps appear, the obtained results show small contribution steps at odd and fractional multiples of the ac frequency, coming from the new features of the dynamical current (see Fig. 2(b) below). Nevertheless, all these contributions are of the order of the overlap between inphase MFs and negligible compared to the height of the even Shapiro steps. Remarkably, we have found a regime where the effect of considering a dominant contribution of extra $2 \pi$ periodic Andreev modes, does not modify the spectrum of even Shapiro steps, providing a robust measurement of the $4 \pi$ periodicity. In addition, we have seen that typical time scales $(\mu \mathrm{s})$ 37] of QP produce a negligible effect on the dynamics of the system.

Ideally, a generic 1D Josephson junction in the presence of Majorana fermions can be described by the MFs placed at the junction, yielding a Hamiltonian

$$
H_{0}=i E_{J} \cos (\varphi / 2) \eta_{1} \eta_{2},
$$




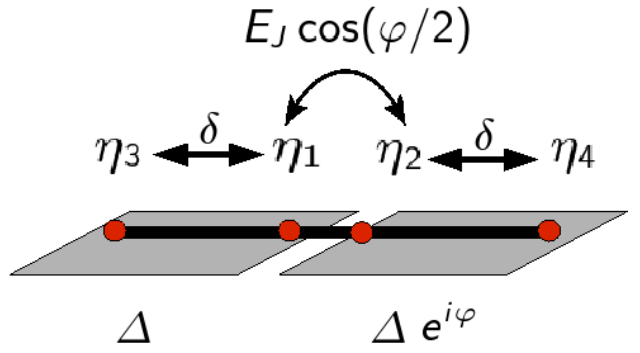

FIG. 1: Josephson junction with a nanowire on top. Red spots (color online) represent Majorana fermions. Double arrows represent the overlap between the Majorana fermions.

where $E_{J}$ is the Josephson energy of the junction, $\varphi$ is the gauge invariant phase difference and the operators $\eta_{i}$ are Hermitian $\eta_{i}=\eta_{i}^{\dagger}$ and they fulfill the anticommutator relation $\eta_{i} \eta_{j}+\eta_{j} \eta_{i}=2 \delta_{i, j}$. Due to the presence of $\mathrm{MF}$ the periodicity of the spectrum is $4 \pi$. In finite systems, in-phase MF may recombine into usual fermions through the overlap of their wave functions [1, 36]. In order to account with this phenomenon an extra term should be added, so that the total Hamiltonian becomes

$$
H=i E_{J} \cos (\varphi / 2) \eta_{1} \eta_{2}+i \delta\left(\eta_{4} \eta_{2}+\eta_{1} \eta_{3}\right),
$$

where we have introduced a parameter $\delta$ to account for overlap between the in-phase MF which decreases exponentially with increasing distance between the Majorana modes (see Fig. 11). Considering that the in-phase MFs are far away compared with those on the junction we will use $\delta \ll E_{J}$. Diagonalizing the Hamiltonian yields the $2 \pi$-periodic energy spectrum (see Fig. 2(a))

$$
E(\varphi)= \pm \sqrt{4 \delta^{2}+E_{J}^{2} \cos ^{2}(\varphi / 2)}
$$

Non-adiabatic changes of the phase leads to transitions between the two eigenstates. Since $E_{J} \gg \delta$, the transition probability is non-vanishing only at the anticrossings of the eigenspectrum, that is, for $\varphi=(2 n+1) \pi$, where $n$ is an integer (see red areas in Fig. 2(a)). Thus, as long as non-adiabatic transitions occur, the overlap between MF is effectively canceled. As a consequence, the $4 \pi$ periodicity in the eigenspectrum, and also in the supercurrent $\left(I \propto \partial_{\varphi} E_{ \pm}\right)$, is recovered. As we will see below the new shape of the current does lead to the expected even steps and also to additional contributions of the order of $\delta$ at odd and fractional multiples of the ac frequency [38].

In order to calculate the transition probability we consider the semiclassical approximation, and we make use of the fact that the velocity at the anticrossings is linear, therefore, transitions between states can be obtained by means of the Landau-Zener probability

$$
P_{L Z}=\exp \left(-2 \pi \frac{4 \delta^{2}}{E_{J} \hbar \dot{\varphi}}\right)
$$

It is important to remark that in the experiment we are analyzing, the phase $\varphi$ is biased by a noisy voltage coming from fixing an external current. These voltage fluctuations are translated to phase fluctuations by the fact that $\dot{\varphi} \propto V$, and thus, dephasing enters into play. We have estimated that the dephasing time $t_{D}$ is much shorter than the time needed to change the phase by $\varphi \rightarrow \varphi+2 \pi$. Therefore, we assume that interference effects can be neglected, and Landau-Zener transitions (LZT) can be considered individually. Coherences between LZTs have been recently analyzed phenomenologically [35], and in more detail [34] for the case of a voltage biased junction, where also additional Andreev levels, QP and inelastic transitions have been considered. However, we would like to stress that the current biased setup analyzed in this letter for the first time has two advantages: contrary to the voltage biased case, it (1) shows a robust signal of small odd integer Shapiro steps even if the case of a multimode wire and (2) the observation is not masked by interference effects as those are absent in our case.

Once we have analyzed the dynamical transitions of the junction, we are ready to include their dynamical effects on the current. To this aim we introduce the function $I_{M}(\varphi)$ in the supercurrent

$$
I(\varphi)=I_{M}(\varphi) \frac{2}{E_{J}} \frac{\partial}{\partial \varphi} E(\varphi) .
$$

The function, $I_{M}(\varphi)$, can take the constant values $\pm I_{M}$, where $I_{M}$ is the maximum value of the supercurrent, which is of the order of nA. During the adiabatic period, the function $I_{M}(\varphi)$ remains constant and whenever there is a LZT, $I_{M}(\varphi)$ changes its sign. To understand the change of the sign we can compare in Fig. 2(b) the adiabatic and non-adiabatic passage through the anticrossings (solid and dashed respectively). After each anticrossing the curve coming from a LZT acquires a negative sign respect to the adiabatic passage. Thus, we describe the dynamical effects on the current produced by the LZT by changing the sign of $I_{M}$.

We study the Shapiro experiment by means of the resistive shunted junction model (RSJ) in the overdamped limit [31, 39]. The induced voltage on the junction can be calculated by solving the differential equation

$$
I_{0}+I_{1} \sin \left(\omega_{a c} t\right)=I(\varphi(t))+\frac{\hbar}{2 e R} \dot{\varphi}(t) .
$$

This equation is obtained from Kirchoff's law where an external DC $I_{0}$ and AC $I_{1} \sin \left(\omega_{a c} t\right)$ currents are applied to the junction. The outgoing current is modeled by a parallel circuit whose components are, $I(\varphi(t))$, given by Eq. (5), and a resistive current $(\hbar / 2 e R) \dot{\varphi}$ originating from the existence of quasiparticles. The solution of the differential equation (6), allows to obtain the induced voltage $V=\hbar \dot{\varphi} / 2 e$. This equation is solved dynamically since $I_{M}(\varphi)$ changes its sign depending on whether the LZT occurs or not. In order to include such a dynamical 


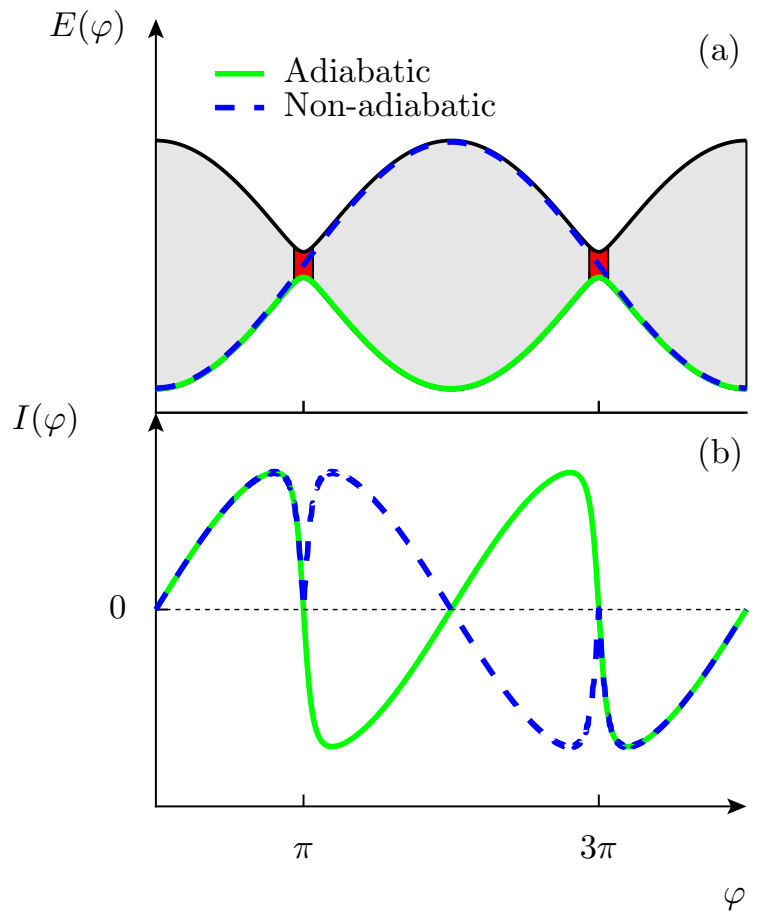

FIG. 2: (Color on line) Eigenspectrum (a) and Andreev current (b) vs $\varphi$, after zero (green solid) and two (blue dashed) LZT occurred. In panel (a) light and dark gray areas (gray and red) correspond to the adiabatic and non-adiabatic evolution respectively. We have represented two different limits of dynamics; the adiabatic limit (green solid line), presenting $2 \pi$ periodicity and the non-adiabatic limit (blue dashed line) presenting $4 \pi$ periodicity.

effect we compute $P_{L Z}$, on the anticrossings. Namely, when $\varphi(t)=(2 n+1) \pi$ we evaluate the phase velocity $\dot{\varphi}$ and compare the resulting $P_{L Z}$ with a random number to determine whether a LZT occurs or not.

In order to perform the calculations closer to the real experiment we have included two additional phenomena: the presence of extra Andreev modes and QP. Majorana fermions may occur in multimode nanowires as long as there is an odd-number of bands occupied [18, 40]. The extra modes contribute with the conventional $2 \pi$-periodic supercurrent, i.e., by means of adding $I_{c} \sin (\varphi)$ to $I(\varphi)$ in Eq. (5). The value $I_{c}$ is a constant parameter whose contribution can be much greater than $I_{M}$, due to the possibility that several modes are occupied. It is worth to mention that we have not considered dynamical effects between the Majorana and Andreev modes due to the fact that the energy difference between them is high enough compared to the phase velocity. This can be assured whenever the transparency of the junction fulfils $T \ll 1[26,34]$. The second effect is the QP, which accounts for the effect of quasiparticles tunneling from the contacts. The change in the number of quasiparticles provokes a transition from one of the eigenstates to the

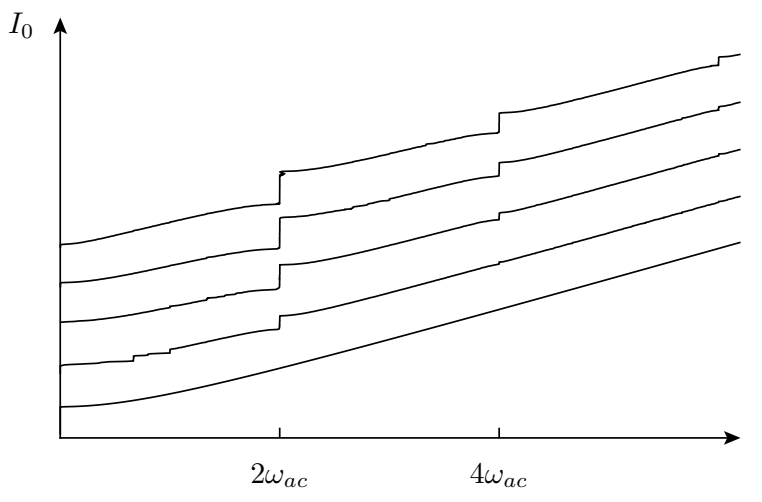

$\langle\dot{\varphi}\rangle$

FIG. 3: Current-Voltage curves for $I_{M}=1 \mathrm{nA}, E_{J} / \delta=500$, $R=3 \mathrm{k} \Omega, \omega_{a c}=10^{10} \mathrm{~Hz}$ and $I_{c}=0 . I_{1}$ takes values from 0 to $4 \mathrm{nA}$ from bottom to top with a step of $1 \mathrm{nA}$. For the sake of clarity all the curves are shifted a constant amount.

other one [5]. These transitions affect the current periodicity, and in principle they should appear reflected on the Shapiro steps positions.

The main results of our calculations are shown in Figs. 3and 4 There, we have represented current-voltage curves for $I_{c}=0$ and $I_{c}=10 I_{M}$ respectively, using $I_{M}=1 \mathrm{nA}$ and $\mathrm{R}=3 \mathrm{k} \Omega$, altogether lead to measure voltages of the order of $V \approx 10 \mu \mathrm{V}$. We have set $E_{J} / \delta=500$, making that $P_{L Z}$ is very close to one at any value of $I_{0}$. Therefore, the supercurrent presents a $4 \pi$ periodicity most of the time, whose form is given by the dashed curve of Fig. 2(b).

In figure 3 we have plotted the $I / V$ curves for $\omega_{a c}=$ $10^{10} \mathrm{~Hz}$ and $I_{1}=0$ up to $I_{1}=4 \mathrm{nA}$ with an increment of $1 \mathrm{nA}$. We can observe that the height of even Shapiro steps dominate the Shapiro spectrum. Thus, revealing clearly the resulting $4 \pi$ periodicity of the junction. Moreover, there are some contributions of $\delta$ order placed at odd and fractional multiples of $\omega_{a c}$, coming from the new form of the supercurrent acquired by the LZT. It is worth to remind that in the ideal case, i.e. $I(\varphi)=I_{M} \sin (\varphi / 2)$ such steps do not appear.

In figure 4 we show the $I / V$ curves in the presence of a large $2 \pi$ contribution, $I_{c}=10 I_{M}$. Different curves correspond to different values of $\omega_{a c}$, in decreasing order from top to bottom. In this situation, one would expect to obtain all integer steps coming from the dominant $2 \pi$ character of the supercurrent. In turn, one observes a progressive reduction of the odd steps as we decrease $\omega_{a c}$ up to $2 e R I_{M} / \hbar$, while even steps hold. We have demonstrated that the reduction of odd steps is caused by the presence of a Majorana mode and can, in principle, be found even in the case where $I_{c} \gg I_{M}$. It is important to remark that this behavior is completely different to the voltage biased experiment, where the $2 \pi$ contribution gives rise to steps at even and odd multiples, with 


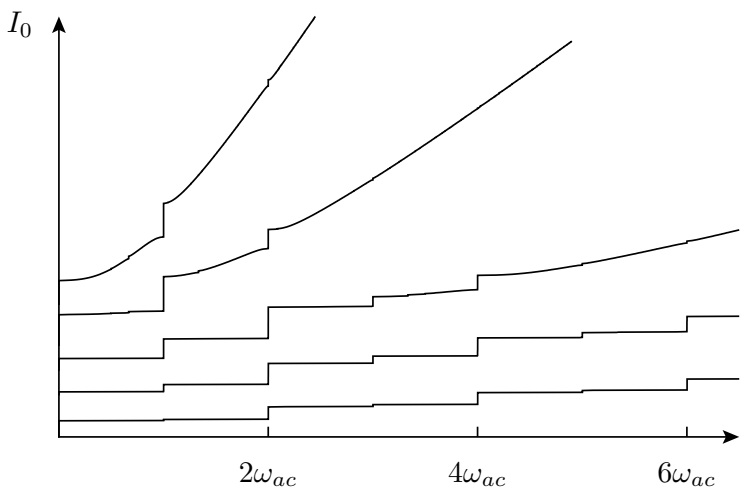

$\langle\dot{\varphi}\rangle$

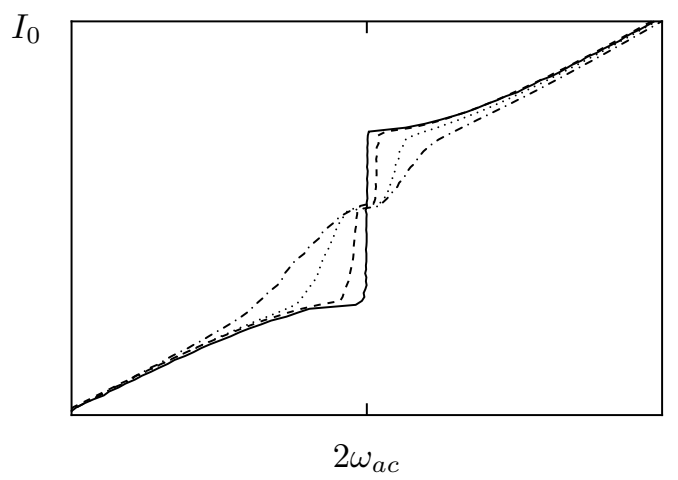

$\langle\dot{\varphi}\rangle$
FIG. 4: Current-Voltage curves for $I_{M}=1 \mathrm{nA}, E_{J} / \delta=500$, $R=3 \mathrm{k} \Omega, I_{1}=3 \mathrm{nA}$ and $I_{c}=10 I_{M}$. The ac frequency $\omega_{a c}$ takes the values: $10^{11}, 5 \times 10^{10}, 2 \times 10^{10}, 10^{10}$ and $5 \times 10^{9} \mathrm{~Hz}$ from top to bottom. We observe a progressive quenching of the odd steps as we decrease $\omega_{a c}$ up to $10^{10} \mathrm{~Hz}$. For the sake of clarity all the curves are shifted down a constant amount.

heights proportional to $I_{c}$ [33]. Thus, when $I_{c} \gg I_{M}$ the detection of the Majorana mode from the Shapiro spectrum will in general complicated.

The observed behavior stands on the non-linear character of the RSJ model versus the linear one of the voltage biased experiment. In order to understand this it is necessary first to revisit the undriven case, i.e. $I_{1}=0$, where both, $2 \pi$ and $4 \pi$ contributions are present. In such situation, we show [38] that due to the non-linear character of the RSJ equation, the presence of the $4 \pi$ contribution imposes a strong $4 \pi$ character to the junction for a range of voltages of the order of $I_{M} R$, even in the case when $I_{c} \gg I_{M}$. Ac currents with frequencies up to $2 e R I_{M} / \hbar=10^{10} \mathrm{~Hz}$, correspond to this range of voltages and thus are expected to show predominately even Shapiro step.

We can extract additional information from the current-voltage curves. It can be demonstrated that the height of the 0 -step at $I_{1}=0$, is approximately equal to $I_{c}+I_{M} / \sqrt{2}$ for $I_{c} \gg I_{M}$. Then, tuning the gate voltage one could, in principle, fill a single extra mode and measure the resulting contribution to the current. Then, one would be able to determine $I_{M}$.

Until now the calculations we have shown were performed using the value $E_{J} / \delta=500$, which leads to $P_{L Z} \simeq 1$ for the whole spectrum of $I_{0}$. Increasing the overlap $\delta$ reduces $P_{L Z}$ making that non-LZT may occur, provoking a departure from the studied $4 \pi$ periodicity of the current. The main changes are produced in the positions of the steps, where we observe that the Shapiro step splits in two (see Fig. 55). In order to shed some light on the numerical results we can average out the position of the non-LZT events and approximate $I_{M}(\varphi)$ by means of a Fourier series [38]. The resulting current-voltage curve
FIG. 5: Current voltage curves for different values of $E_{J} / \delta=500$ (solid), 100 (dashed), 50 (dotted) and 30 (dotteddashed), $I_{1}=1 \mathrm{nA}$, the rest of the parameters are taken from those used in Fig. 3. We show the splitting of a Shapiro step by the increment of the overlap $\delta$

behaves rather similar to the numerical results obtained by means of the stochastic model presented here. Comparing both methods we extract that the splitting is of the order of $\left(1-P_{L Z}\right) / 2$. We observe that the Fourier approximation fails whenever we decrease $E_{J} / \delta$ up to 30 .

Quasiparticle poisoning relaxation time is typically of the order of $\mu \mathrm{s}$ [37]. For the ac driving frequencies considered in our work, $\omega_{a c} \approx 10^{10} \mathrm{~Hz}, \mathrm{QP}$ does not affect our results significantly.

In conclusion, we have studied the current-biased Shapiro experiment in a finite 1D Josephson junction. We have seen that the effects coming from the finiteness of the 1D system are dynamically decoupled driving the phase by means of an external current. For that purpose, we have analyzed the periodicity of the junction by solving an equation of motion coming from the resistive shunted junction model. We have calculated two different cases, when there are no extra $2 \pi$ modes, i.e. $I_{c}=0$, we have obtained that we can always determine the presence of the Majorana mode due to the appearance of steps at even multiples of the ac frequency $\omega_{a c}$ (see Fig. 3). In turn, when $I_{c} \gg I_{M}$, we have found that thanks to the non-linear effects coming from the dynamics of RSJ, the junction behaves $4 \pi$ periodically for a range of voltages of the order $I_{M} R$. We have found that it is possible to match this range, and therefore its $4 \pi$ behavior, by using ac frequencies of the order of $\omega_{a c} \lesssim 2 e R I_{M} / \hbar=10^{10} \mathrm{~Hz}$ (see Fig. (4). The resulting Shapiro steps are thus placed at even multiples of the frequency $\omega_{a c}$. In addition, we have seen that the effects of QP on the current are negligible at the typical estimated timescales. From our results, we believe that performing the current-biased Shapiro experiment is a strong tool to prove the existence of Majorana fermions in finite nanowires. 


\section{ACKNOWLEDGMENTS}

We acknowledge R. Hussein for his invaluable help in improving the numerical codes. We acknowledge L. P. Kouwenhoven and S. Frolov for enlightening discussions. One of the authors (F.D) acknowledges the hospitality of the Kavli Institute of Nanoscience Delft. We also acknowledge financial support from Grant No. MAT201124331 and from ITN, Grant No. 234970 (EU). FH is grateful for support from the Alexander von Humboldt foundation.

[1] G. E. Volovik and V. M. Yakovenko, J. Phys. 1, 5263 (1989).

[2] G. Moore and N. Read, Nuc. Phys. B 360, 362 (1991).

[3] N. Read and D. Green, Phys. Rev. B 61, 10267 (2000).

[4] A. Y. Kitaev, Ann. Phys. 303, 2 (2003).

[5] L. Fu and C. L. Kane, Phys. Rev. Lett. 100, 096407 (2008).

[6] R. M. Lutchyn, J. D. Sau, and S. D. Sarma, Phys. Rev. Lett. 105, 077001 (2010).

[7] Y. Oreg, G. Refael, and F. von Oppen, Phys. Rev. Lett. 105, 177002 (2010).

[8] T.-P. Choy, J. M. Edge, A. R. Akhmerov, and C. W. J. Beenakker, Phys. Rev. B 84, 195442 (2011).

[9] D. A. Ivanov, Phys. Rev. Lett. 86, 268 (2001).

[10] A. Nayak, S. H. Simon, A. Stern, M. Friedman, and S. D. Sarma, Phys. Rev. Mod. 80, 1083 (2008).

[11] J. Linder, Y. Tanaka, T. Yokoyama, A. Sudbø, and N. Nagaosa, Phys. Rev. Lett. 104, 067001 (2010).

[12] V. Mourik, K. Zuo1, S. M. Frolov, S. R. Plissard, E. P. A. M. Bakkers, and L. P. Kouwenhoven, ArXiv 1204.2792v1, (2012).

[13] J. G. Rodrigo, V. Crespo, H. Suderow, S. Vieira, and F. Guinea, ArXiv 1203.5265v1, (2012).

[14] L. P. Rokhinson, X. Liu, and J. K. Furdyna, ArXiv 1204.4212v1, (2012).

[15] C. Bolech and E. Demler, Phys. Rev. Lett. 98, 237002 (2007).

[16] K. T. Law, P. A. Lee, and T. K. Ng, Phys. Rev. Lett. 103, 237001 (2009).

[17] M. Wimmer, A. R. Akhmerov, J. P. Dahlhaus, and C. W. J. Beenakker, New J. Phys. 13, 053016 (2011).

[18] M. Wimmer, A. R. Akhmerov, M. V. Medvedyeva, J. Tworzydlo, and C. W. J. Beenakker, Phys. Rev. Lett. 105, 046803 (2010).

[19] A. R. Akhmerov, J. P. Dahlhaus, F. Hassler, M. Wimmer, and C. W. J. Beenakker, Phys. Rev. Lett. 106, 057001 (2011)

[20] K. Flensberg, Phys. Rev. B 82, 180516(R) (2010).

[21] J. Nilsson, A. R. Akhmerov, and C. W. J. Beenakker, Phys. Rev. Lett. 101, 120403 (2008).

[22] L. Fu, Phys. Rev. Lett. 104, 056402 (2010).

[23] C. Benjamin and J. K. Pachos, Phys. Rev. B 81, 085101 (2010).

[24] A. Y. Kitaev, Phys. Usp. 44, 131 (2001).

[25] H. J. Kwon, K. Sengupta, and V. M. Yakovenko, Eur. Phys. J. B 37, 349 (2003).

[26] L. Fu and C. L. Kane, Phys. Rev. B 79, 161408(R)
(2009).

[27] Y. Tanaka, T. Yokoyama, and N. Nagaosa, Phys. Rev. Lett. 103, 107002 (2009).

[28] P. A. Ioselevich and M. V. Feigel'man, Phys. Rev. Lett. 106, 077003 (2011).

[29] K. T. Law and P. A. Lee, Phys. Rev. B 84, 081304 (2011).

[30] B. van Heck, F. Hassler, A. R. Akhmerov, and C. W. J. Beenakker, Phys. Rev. B 84, 180502(R) (2011).

[31] M. Tinkham, Introduction to superconductivity, International series in pure and applied physics (McGraw-Hill, Inc, New York, 1996).

[32] S. Shapiro, Phys. Rev. Lett. 11, 80 (1963).

[33] L. Jiang, D. Pekker, J. Alicea, G. Refael, Y. Oreg, and F. von Oppen, Phys. Rev. Lett. 107, 236401 (2011).

[34] P. San-Jose, E. Prada, and R. Aguado, ArXiv 1112.5983, (2011).

[35] D. I. Pikulin and Y. V. Nazarov, Arxiv 1112.6368, (2011).

[36] D. I. Pikulin and Y. V. Nazarov, JETP Lett. 94, 752 (2011).

[37] L. Sun et al., ArXiv 1112.2621, (2011).

[38] See supplementary material for the details.

[39] P. Russer, J. App. Phys. 43, 2008 (1972).

[40] A. C. Potter and P. A. Lee, Phys. Rev. Lett. 105, 227003 (2010). 


\section{Supplementary Material to 'On the dynamical detection of Majorana fermions in finite one-dimensional systems'}

\section{Effective Hamiltonian and eigenvalues of a finite Josephson junction}

We start from a more general Hamiltonian than that given in Eq. (2) of the manuscript. This kind of generic Hamiltonian has been already used previously, to describe a wire in the presence of Majorana fermions overlapping between each other. See for example Refs. 1 or 2 . We then write,

$$
H=i \epsilon \eta_{1} \eta_{2}+i \delta_{R} \eta_{4} \eta_{2}+i \delta_{L} \eta_{1} \eta_{3}
$$

In contrast to Eq. (2), we assume that the overlap $\delta_{L, R}$ between the different sides of the junction can be in general different, which is something that is more similar to a real system. Besides, we simplify the notation by using $\epsilon=E_{J} \cos (\varphi / 2)$. In this situation it is useful to switch to a representation where two Majorana fermions are combined to form one ordinary fermion. Thus, performing the substitutions $\eta_{1}=i\left(l^{\dagger}-l\right), \eta_{2}=r^{\dagger}+r, \eta_{3}=l^{\dagger}+l$, and $\eta_{4}=i\left(r^{\dagger}-r\right)$, we obtain

$$
H=2 \delta_{R}\left(r^{\dagger} r-\frac{1}{2}\right)+2 \delta_{L}\left(l^{\dagger} l-\frac{1}{2}\right)+\epsilon\left(-l^{\dagger} r^{\dagger}-l^{\dagger} r+l r^{\dagger}+l r\right),
$$

where $l^{\dagger} l$ and $r^{\dagger} r=0,1$ counts the occupation of the corresponding state. We rewrite the former Hamiltonian in the base of occupation $\left|n_{L}, n_{R}\right\rangle$, that is $|0,0\rangle,|0,1\rangle,|1,0\rangle$ and $|1,1\rangle$, yielding the matrix

$$
H=\left(\begin{array}{cccc}
-\delta_{L}-\delta_{R} & 0 & 0 & -\epsilon \\
0 & \delta_{R}-\delta_{L} & -\epsilon & 0 \\
0 & -\epsilon & \delta_{L}-\delta_{R} & 0 \\
-\epsilon & 0 & 0 & \delta_{L}+\delta_{R}
\end{array}\right) .
$$

We diagonalize the resulting Hamiltonian and obtain 4 eigenvalues

$$
\begin{aligned}
& E_{ \pm}^{e}= \pm \sqrt{\epsilon^{2}+\left(\delta_{L}+\delta_{R}\right)^{2}} \text { and } \\
& E_{ \pm}^{o}= \pm \sqrt{\epsilon^{2}+\left(\delta_{L}-\delta_{R}\right)^{2}} .
\end{aligned}
$$

where the super index denotes even (e) and odd (o) fermion parity respectively. Assuming that the parity is conserved we can choose one of the eigenvalues and make Landau-Zener transitions between the \pm eigenvalues. In fact, we are only interested in the generic features which an avoided crossing has on the Shapiro steps. Thus we replace either $\left(\delta_{L} \pm \delta_{R}\right)^{2}$ by a characteristic/generic value $2 \delta$ which directly leads to Eq. (3). We have included a footnote in the manuscript making this explicit. Note furthermore that the signs of $\delta_{L / R}$ are random, i.e., determined by microscopic details. Thus, it is not true that the odd sector has a smaller gap than the even sector as one might naively expect when looking at the equations above.

\section{Dephasing produced biasing the phase by means of a noisy voltage}

In the main text we have used the approximation of neglecting interference effects coming from having two or more non-adiabatic transitions [3]. In this appendix we justify this approximation and estimate the dephasing rate produced by biasing the phase difference by means of a fluctuating voltage. For the sake of simplicity we restrict the analysis to the infinite length 1D Josephson junction which in the pseudo-spin basis is given by

$$
H=\frac{E_{J}}{2} \cos (\varphi / 2) \sigma_{z},
$$

where $\sigma_{z}$ denotes the $z$-Pauli matrix. As we have explained, the effect of fixing the current produces thermal fluctuations in the voltage, therefore fluctuations on the phase difference arise around some fixed value $\varphi_{0}$, namely $\varphi(t)=\varphi_{0}+\delta \varphi(t)$. Then, we assume that these fluctuations are small compared with $\varphi_{0}$, so we can rewrite the Hamiltonian approximately

$$
H \approx \frac{E_{J}}{2}\left(\cos \left(\varphi_{0} / 2\right) \sigma_{z}-\delta \varphi(t) \sin \left(\varphi_{0}\right) \sigma_{z}\right)
$$


Thus, the energy difference between the states fluctuates in time. In order to see the effects of these fluctuations one can take a coherent superposition such as

$$
\frac{1}{\sqrt{2}}(|+\rangle+|-\rangle)
$$

where $| \pm\rangle$ are the eigenstates of $\sigma_{z}$. The state at time $t$ is given by

$$
\frac{1}{\sqrt{2}}\left(e^{-i(\Delta t+\phi(t))}|+\rangle+e^{i(\Delta t+\phi(t))}|-\rangle\right) .
$$

Where we have used $\Delta$ to denote the constant energy difference between the states $| \pm\rangle$. The phase $\phi(t)$ accounts the time integral over the fluctuating component of the energy difference

$$
\phi(t)=\frac{E_{J} \sin \left(\varphi_{0}\right)}{\hbar} \int_{0}^{t} d \tau \delta \varphi(\tau) .
$$

These fluctuations are seen in the expectation value

$$
\left\langle\sigma_{x}\right\rangle(t)=\cos (\Delta t+\phi(t)) .
$$

Taking the average over the fluctuating phase we observe a decay of the oscillations

$$
\left\langle\left\langle\sigma_{x}\right\rangle(t)\right\rangle=\cos (\Delta t) e^{-\frac{1}{2}\left\langle\phi(t)^{2}\right\rangle} .
$$

where

$$
\left\langle\phi(t)^{2}\right\rangle=\int_{0}^{t} d t_{1} \int_{0}^{t} d t_{2}\left\langle\delta \varphi\left(t_{1}\right) \delta \varphi\left(t_{2}\right)\right\rangle,
$$

is the time integral of the autocorrelation function. Obtaining this integral allows us to determine lifetime of the coherences. In order to calculate it we have first to relate phase and voltage autocorrelation functions through the Josephson formula

$$
\frac{d}{d t} \varphi(t)=\frac{2 e}{\hbar} V
$$

So that we can express the phase correlation function in terms of the voltage correlation function can be written as

$$
\left\langle\phi(t)^{2}\right\rangle=\left(\frac{2 e}{\hbar}\right)^{2} \int_{0}^{t} d t_{1} \int_{0}^{t} d t_{2} \int_{0}^{t_{1}} d t^{\prime} \int_{0}^{t_{2}} d t^{\prime \prime}\left\langle V\left(t^{\prime}\right) V\left(t^{\prime \prime}\right)\right\rangle .
$$

Since the voltage fluctuations fulfill the Nyquist theorem, we can write

$$
R=\frac{1}{k_{B} T} \int_{-\infty}^{\infty}\langle V(t) V(t+\tau)\rangle d \tau
$$

where $\mathrm{T}$ is the temperature that affects to the external circuit, and $\mathrm{R}$ is its corresponding resistance. Leading to the result

$$
\left\langle\left\langle\sigma_{x}\right\rangle(t)\right\rangle=\cos (\Delta t) e^{-\frac{1}{2} \gamma_{D} t^{3}}
$$

where we have used

$$
\gamma_{D}=\frac{1}{2}\left(\frac{E_{J}}{\hbar}\right)^{2}\left(\frac{2 e}{\hbar}\right)^{2} R k_{B} T .
$$

Due to the fact that the time needed to vary the phase by $\varphi \rightarrow \varphi+2 \pi$ is of the order of $1 / \omega_{a c}$ and $\omega_{a c}=10^{10} \mathrm{~Hz}$, and taking into account that for the parameters that we have considered (see caption of Fig. 3 in the main text) $\gamma_{D} / \omega_{a c}^{3} \gg 1$, coherences become rapidly quenched. 


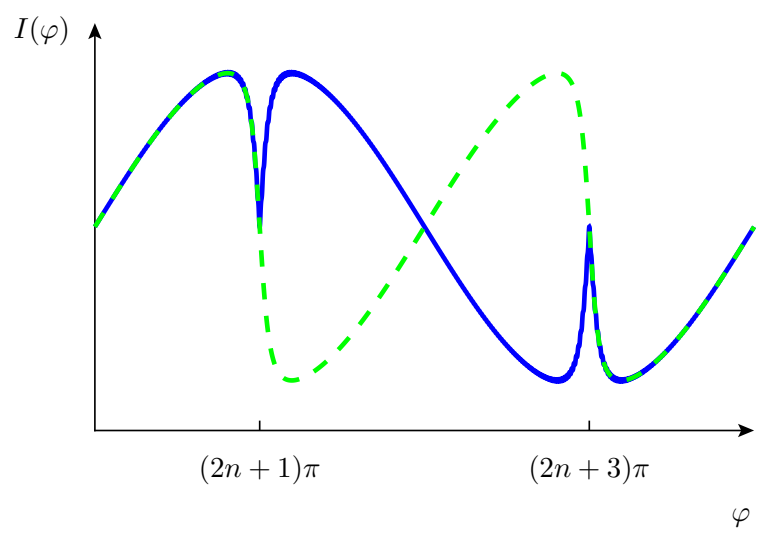

FIG. 6: Dynamical $4 \pi$ current (solid) in a Josephson junction after having two consequtive LZT. Dahsed curve corresponds to the opposite case, i.e. two consequtive non-LZT.

\section{Splitting of the Shapiro steps as a function of $P_{L Z}$}

\section{Fourier decomposition of the $4 \pi$-periodic current}

The current that results of having LZT has the form of the continuous line shown in Fig. 6. However, from time to time the LZT does not occur, thus, the current suffers changes in its periodicity. The consequences of varying the periodicity of the current is reflected on the Shapiro steps pattern. It leads to the splitting of the steps see Fig 7 . In order to have some analytical insight to this phenomena, we use a Fourier expansion of the current and of the non-LZT.

We begin calculating the Fourier components of the current when LZT are always given. We make an odd expansion leading to the terms

$$
I_{F}(\varphi)=\sum_{n=0}^{\infty} b_{n} \sin \left(\frac{n}{2} \varphi\right)
$$

where the Fourier components are given by

$$
b_{n}=\frac{1}{\pi} \int_{0}^{2 \pi} d \varphi I(\varphi) \operatorname{Sgn}[\cos (\varphi / 2)] \sin \left(\frac{n}{2} \varphi\right),
$$

here Sgn denotes the sign function and the current $I(\varphi)$ is given by

$$
I(\varphi)=\frac{\partial}{\partial \varphi} E(\varphi)=\frac{E_{J}^{2} \sin (\varphi)}{8 \sqrt{4 \delta^{2}+E_{J}^{2} \cos ^{2}(\varphi / 2)}} .
$$

where $\delta$ is the overlap between the in-phase MF and $E_{J}$ is the Josephson energy. The integral is zero for $n=\dot{2}$ yielding just the odd Fourier contributions. Taking into account that $\delta \ll E_{J}$, we take the first order (Taylor) expanssion in $\delta$, yielding

$$
\begin{aligned}
& b_{0}=\frac{E_{J}}{4}-\frac{2 \delta}{\pi} \\
& b_{n}=(-1)^{n+1} \frac{2 \delta}{\pi} .
\end{aligned}
$$

This leads to write the current as,

$$
I_{F}(\varphi)=\frac{E_{J}}{4} \sin (\varphi / 2)+\frac{2 \delta}{\pi} \sum_{n=0}^{\infty}(-1)^{n+1} \sin \left(\frac{2 n+1}{2} \varphi\right) .
$$

As expected, the current has a $4 \pi$ contribution proportional to $E_{J}$ perturbed by a sum of extra terms which are of the order of $\delta$. 


\section{Non-Landau Zener Transitions}

We represent the phenomenon of not having LZT in a different way as it is presented in the main text. There, having a LZT was simulated by a change of sign. Indeed, this has been already included in the Fourier expansion above eq. 30, and for this reason here having a non-LZT will be simulated by changing as well the sign of the current. To this aim we will use a unit-step that changes its sign after a certain period, which depends on the average of the LZ probability.

In the calculations we perform in the main text, this effect is estimated stochastically. In turn, here we consider the average of a given LZT and non-LZT. Since a LZT is not affected by a previous phase, this approximation will be in principle accurate. Thus, we consider the averaged LZ probability $P_{L Z}$, namely, in average after a number LZT there is a non-LZT. The next approximation will be to consider that these non-LZT are distributed homogenously in time. This approximation, is a bit more fragile, due to the fact that we are measuring the periodicity of the current by the position of the Shapiro steps. Thus, to consider a homogeneous distribution or an inhomogeneous one will lead to different results in the experiment. In principle, the homogeneous distribution will be only appropiated when the non-LZT are separated enough in time, so that the periodicity of the $4 \pi$ current (eq. 30) dominates.

In addition, the function has to include the fact that the change of sign can only come at certain values of the phase, i.e. when $\varphi=(2 n+1) \pi$. Then, it is straightforward to obtain the period as

$$
T=2 \pi(2 P)
$$

where

$$
P=\operatorname{Integer}\left[\frac{1}{1-P_{L Z}}\right]
$$

and therefore the Fourier expansion becomes

$$
\operatorname{NLZ}(\varphi)=\sum_{i=0}^{\infty} \frac{4}{(2 i+1) \pi} \sin \left(\frac{2 i+1}{2 P}(\varphi+\pi)\right)
$$

\section{Total current}

Then, taking the product of the Fourier expansions of the current and the change of sign and we arrive to the expression

$$
\begin{aligned}
I(\varphi)= & \sum_{i=0}^{\infty} \frac{E_{J}}{2(2 i+1) \pi}\left\{\cos \left(\left(\frac{1}{2}-\frac{2 i+1}{2 P}\right) \varphi\right)-\cos \left(\left(\frac{1}{2}+\frac{2 i+1}{2 P}\right) \varphi\right)\right\} \\
& +\frac{\delta}{\pi} \sum_{i=0}^{\infty} \frac{4}{(2 i+1) \pi} \sum_{n=0}^{\infty}\left\{(-1)^{n+1}\left[\cos \left(\left(\frac{2 n+1}{2}-\frac{2 i+1}{2 P}\right) \varphi\right)-\cos \left(\left(\frac{2 n+1}{2}+\frac{2 i+1}{2 P}\right) \varphi\right)\right]\right\} .
\end{aligned}
$$

We can observe that due to the new periodicity the former $4 \pi$ and the rest of the Fourier components, are splitted in two by a quantity proportional to $2\left(1-P_{L Z}\right)$, as occurs in the numerics (see Fig. 7). We can also see that the average model deviates from the stochastic calculations as we increase $\delta$. This is caused because increasing $\delta$, the number of non-LZT increases so that non-LZT may not occur isoletely, changing thus the periodicity. Therefore, for lower LZ probabilities the average between the pure $2 \pi$ and $4 \pi$ currents weighted by $P_{L Z}$ give more accurate results.

It is worthy to remark that the Fourier current presented above, has been developed to show analyticaly the splitting, however, numerical results of the averaged model have been obtained from the current given by

$$
I(\varphi)=I_{M} \frac{2}{E_{J}} \frac{\partial}{\partial \varphi} E(\varphi) \operatorname{Sgn}\{\cos (\varphi / 2)\} \operatorname{Sgn}\left\{\cos \left(\frac{\varphi-(P-1) \pi}{2 P}\right)\right\}
$$

\section{Shapiro experiment: Robustness of the even steps}

The Shapiro experiment has been proposed [1, 4, 6] to detect the presence of Majorana fermions because it allows to deduce the periodicity of the current-phase relation of the junction, and therefore to distinguish between Majorana and normal modes, whose current is proportional to $\sin (\varphi / 2)$, and $\sin (\varphi)$ respectively. 
$I_{0}$

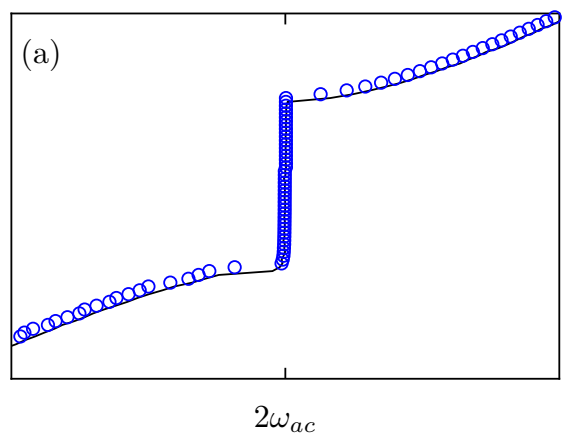

$\langle\dot{\varphi}\rangle$

$I_{0}$

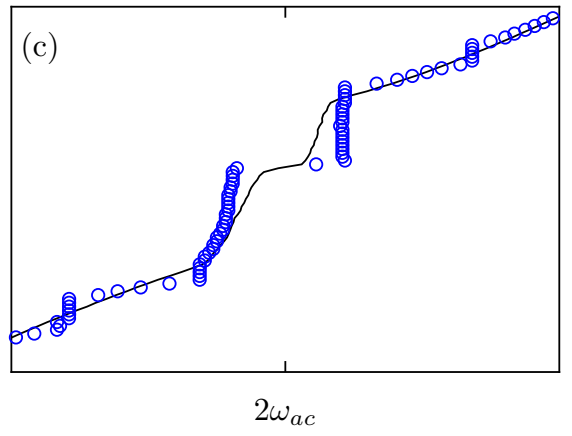

$I_{0}$

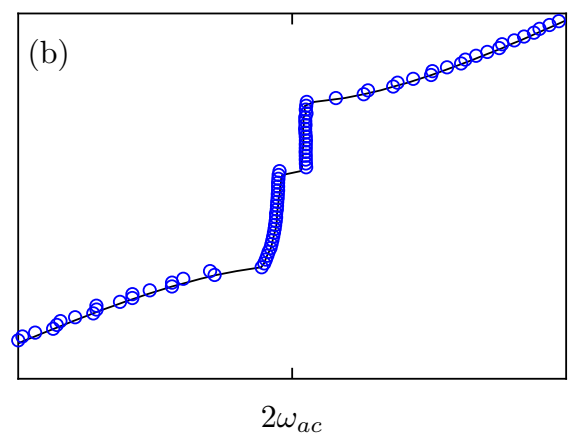

$\langle\dot{\varphi}\rangle$

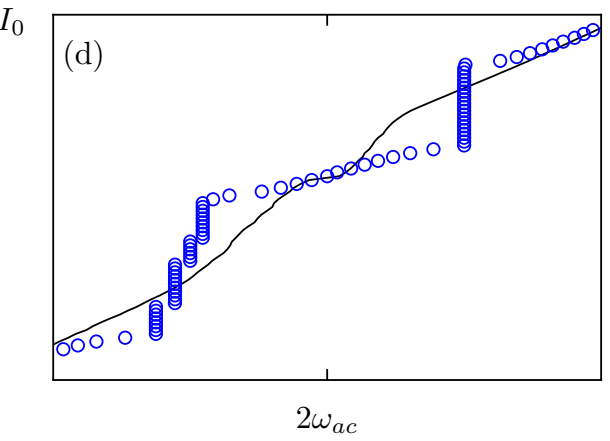

$\langle\dot{\varphi}\rangle$

$\langle\dot{\varphi}\rangle$

FIG. 7: Current/voltage curves for different values of $E_{J} / \delta$ : (a) 500 , (b) 100 , (c) 50 and (d) $30, I_{1}=1 \mathrm{nA}$, the rest of the parameters are taken from those used in Fig 3 in the main text. Solid lines are the values obtained by means of the stochastic model while blue circles come from the averaged model presented here.

The physical fundament of the experiment consists of a resonance phenomena which involves the natural frequency of the Majorana (normal) Josephson junction $\omega_{0}=(2) \mathrm{eV} / \hbar$ and the frequency of an applied rf current (voltage) $\omega_{a c}$. When the resonance is fulfilled, i.e. $V=n \hbar \omega_{a c} /(2) e$, Shapiro steps arise. We can now see that Shapiro steps coming from a Majorana mode arise just at even multiples of the applied ac frequency, while normal modes appear at the whole spectrum of integer multiples, see Fig. 8 .

In practice, to distinguish the presence of a Majorana mode is expected to be more complicated due to the presence of both, normal and Majorana modes, presenting a higher contribution the former one. In this scenario, Shapiro steps should appear at even and odd positions. However, in the main text we have found a regime in the current biased experiment where the Majorana mode can give rise to even steps in the presence of a much higher $2 \pi$ contribution to the current. This section is dedicated to explain the reason of this behavior.

The structure of this section consists of two parts. In the first one we present briefly the voltage biased experiment, while in the second we analyze in more extension the current biased experiment. The second part is divided in two subsections where we analyze the numerical results of the RSJ model in the absence and presence of Majorana and with and without ac current.

\section{Voltage biased Shapiro experiment}

In the presence of Majorana and normal modes the Josephson current is given by

$$
I(\varphi)=I_{M} \sin (\varphi / 2)+I_{c} \sin (\varphi),
$$

where $I_{M} \ll I_{c}$. On the other hand, the phase difference is given by the Josephson formula

$$
\frac{d}{d t} \varphi=\frac{2 e}{\hbar} V(t)
$$



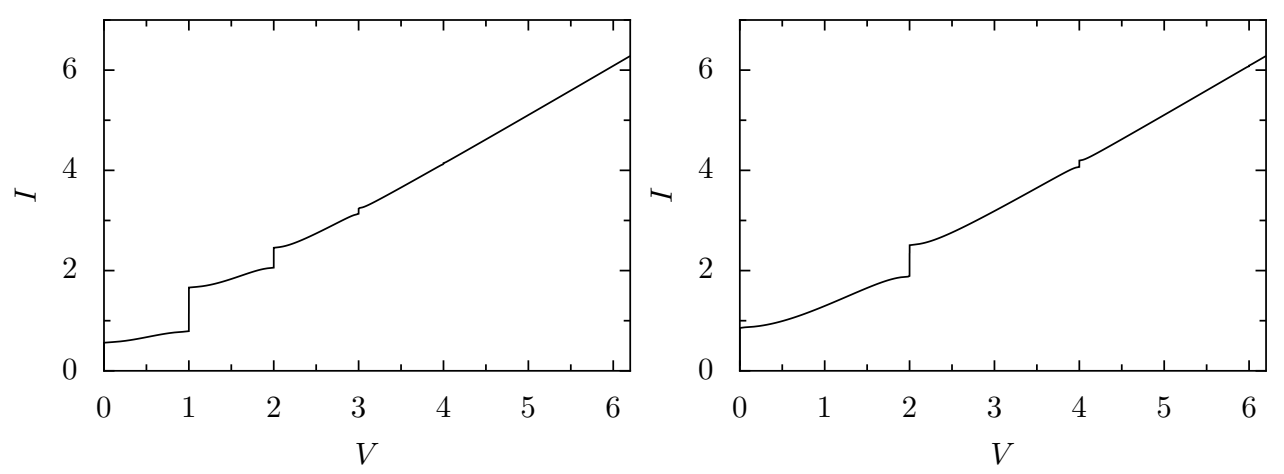

FIG. 8: $I / V$ curve of a current biased Shapiro experiment in the presence of normal (left) and Majorana modes (right).

This means that applying an external ac-voltage

$$
V(t)=V_{0}+V_{1} \cos \left(\omega_{a c} t\right),
$$

we will be able to tune the phase difference, leading to

$$
\varphi(t)=\varphi_{0}+\omega_{0} t+\frac{2 e V_{1}}{\hbar} \sin \left(\omega_{a c} t\right)
$$

Here we have used $\omega_{0} \equiv 2 e V_{0} / \hbar$. Then, we substitute eq. (39) into the Josephson current eq. (36)

$$
I(t)=I_{M} \sum_{n}(-1)^{n} J_{n}\left(\frac{e V_{1}}{\hbar \omega_{a c}}\right) \sin \left(\frac{\varphi_{0}}{2}+\left(\frac{\omega_{0}}{2}-n \omega_{a c}\right) t\right)+I_{c} \sum_{n}(-1)^{n} J_{n}\left(\frac{2 e V_{1}}{\hbar \omega_{a c}}\right) \sin \left(\varphi_{0}+\left(\omega_{0}-n \omega_{a c}\right) t\right) .
$$

Thus, in the stationary limit just the contributions with $n \omega_{a c}=\omega_{0}$ survive, namely

$$
\bar{I}=I_{M} \sum_{n}(-1)^{n} J_{n}\left(\frac{e V_{1}}{\hbar \omega_{a c}}\right) \delta\left(\left(\omega_{0} / 2-n \omega_{a c}\right)\right)+I_{c} \sum_{n}(-1)^{n} J_{n}\left(\frac{2 e V_{1}}{\hbar \omega_{a c}}\right) \delta\left(\left(\omega_{0}-n \omega_{a c}\right)\right) .
$$

with Dirac deltas placed at integer values of the radio frequency, $n \omega_{a c}$. It has to be noted that the contribution of the normal and Majorana modes is linear in $I_{c, M}$, and for this reason the height of the even steps will be sligthly modified by the presence of the Majorana mode in the case that $I_{c} \gg I_{M}$. Therefore, we can understand that the presence of the normal Andreev modes, which contributes with steps placed at all integer multiples, will difficult the separation of both contributions and thus the identification of the Majorana mode. For this reason, in the situation where $I_{c} \gg I_{M}$, the voltage biased experiment seems to be a non-sensitive method to detect the Majorana mode.

\section{Current biased Shapiro experiment}

In the main text we have performed numerical calculations for the current biased Shapiro experiment. We have presented I-V curves for different values of $\omega_{a c}$ and seen that for some special regime of frequencies odd steps vanish, pointing out the $4 \pi$ periodicity of the junction. In this section we give numerical arguments to explain this phenomena. In order to simplify the discussion we will study the ideal case, where the Majorana fermions at the extremes of the quantum wire are infinitely apart from the junction, so that the overlap is zero and the supercurrent becomes proportional to $\sin (\varphi / 2)$. In this situation the equation under study is

$$
I_{0}+I_{1} \sin \left(\omega_{a c} t\right)=I(\varphi(t))+\frac{\hbar}{2 e R} \dot{\varphi}(t) .
$$

This equation is obtained from Kirchoff's law where an external dc $I_{0}$ and ac $I_{1} \sin \left(\omega_{a c} t\right)$ currents are applied to the junction. The outgoing current is modeled by a parallel circuit whose components are, $I(\varphi(t))$, given by Eq. (36), and a resistive current $\hbar /(2 e R) \dot{\varphi}$ originating from the existence of quasiparticles. 


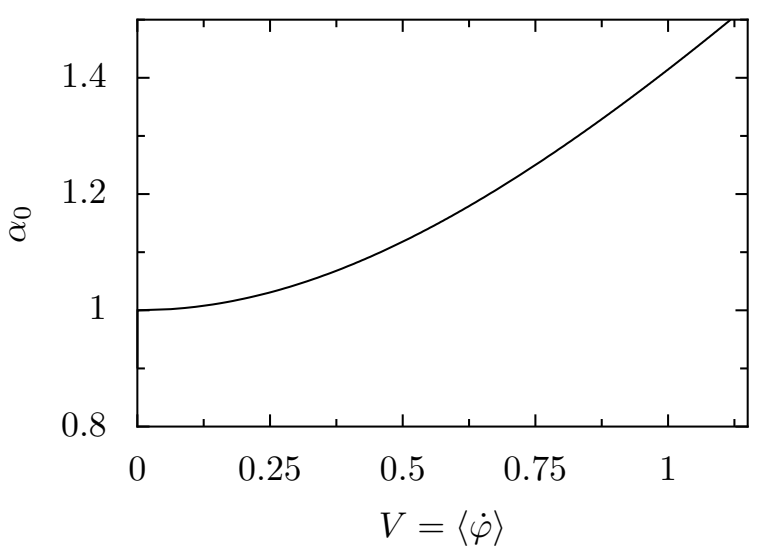

FIG. 9: $\alpha_{0}$ vs $\langle\dot{\varphi}\rangle$ in the absence of the Majorana mode and the ac current.

In order to analyze the equation it results convenient to renormalize the involved parameters. Therefore, we divide the entire equation by $I_{c}$, and transform the time to a dimensionless quantity $\tau=(2 e R / \hbar) t$. This provokes a renormalization on the ac frequency $\xi=\hbar \omega_{a c} / 2 e R I_{c}$, leading to rewrite the equation as

$$
\dot{\varphi}(\tau)=\alpha_{0}+\alpha_{1} \sin (\xi \tau)-\sin (\varphi)-\alpha_{M} \sin (\varphi / 2)
$$

where the renormalized intensities are given by $\alpha_{i}=I_{i} / I_{c}$. Now, the analysis of the equation is reduced to the study the parameter regime of $\alpha_{1}, \alpha_{M}$, and $\xi$.

The analytic solution of the equation in the absence of the Majorana mode is not known, and only approximate solutions have been obtained [7 9], although none of them are valid in the regime where we observe that odd steps vanish. Besides, the $4 \pi$ term coming from the presence of the Majorana mode makes the system even more complicated. For all these reasons, it is out of the scope of this appendix to try to give an analytical insigth of the differential equations. In turn, we explore the numerical solutions and explain its general behavior.

\section{Undriven system without the Majorana term}

The equation of motion of the system without neither MF nor ac current is given by

$$
\dot{\varphi}(t)=\alpha_{0}-\sin (\varphi(t)) .
$$

In Fig. 9 we show the dependence of $\langle\dot{\varphi}\rangle$ as a function of $\alpha_{0}$. This case is analytically solvable, and the average of the voltage is given by

$$
\bar{V}=\langle\dot{\varphi}\rangle=\sqrt{\alpha_{0}-1} .
$$

This equation is only valid for $\alpha_{0}>1$. The value $\alpha_{0}=1$ is called the critical value, which we denote by $\alpha_{c}$, and is defined as the value up to which the induced voltage passes from zero to a finite value. In general, this value will change depending on whether we add the Majorana mode and/or the ac current. This value will be important in the incoming analysis.

In panel a of Figs. 10 and 11 we show numerical results of $\varphi(\tau)$ and $\dot{\varphi}(\tau)$ as a function of time for two different values of $\alpha_{0}$. Figure 10 presents values of $\alpha_{0}$ close to the critical value of each system, while Fig. 11 presents a higher value of $\alpha_{0}$. As we can observe, $\varphi(\tau)$ presents two different ranges of slopes, i.e. velocities, fast and slow. The slow range is given when the term $\sin (\varphi)=1$, so that the difference $\alpha_{0}-\sin (\varphi)$ is minimum. This happens when $\varphi=(4 n+1) \pi / 2$ (dashed lines of panels a and b of Figs. 10 and 11). In figure 10 $\alpha_{0}$ is very close to $\alpha_{c}$, thus the difference $\alpha_{0}-\sin (\varphi) \rightarrow 0$, and therefore we find a flat slope. For larger values of $\alpha_{0}$ (see Fig. 11) the difference does not tend to zero and we observe an increment of the slope between stairs. In the bottom plot of Figs. 10] and 11 we show results of $\dot{\varphi}$ vs. time. There we can observe that the increment of $\alpha_{0}$ induces a widening of the peaks. On the other hand, the fast range is given for the rest of the values of $\varphi(\tau)$, having a maximum speed when $\sin (\varphi(\tau))=-1$ which occurs at $\varphi=(4 n+3) \pi / 2$. These ranges are periodically repeated with the frequency

$$
\omega_{0}=\bar{V} \text {. }
$$



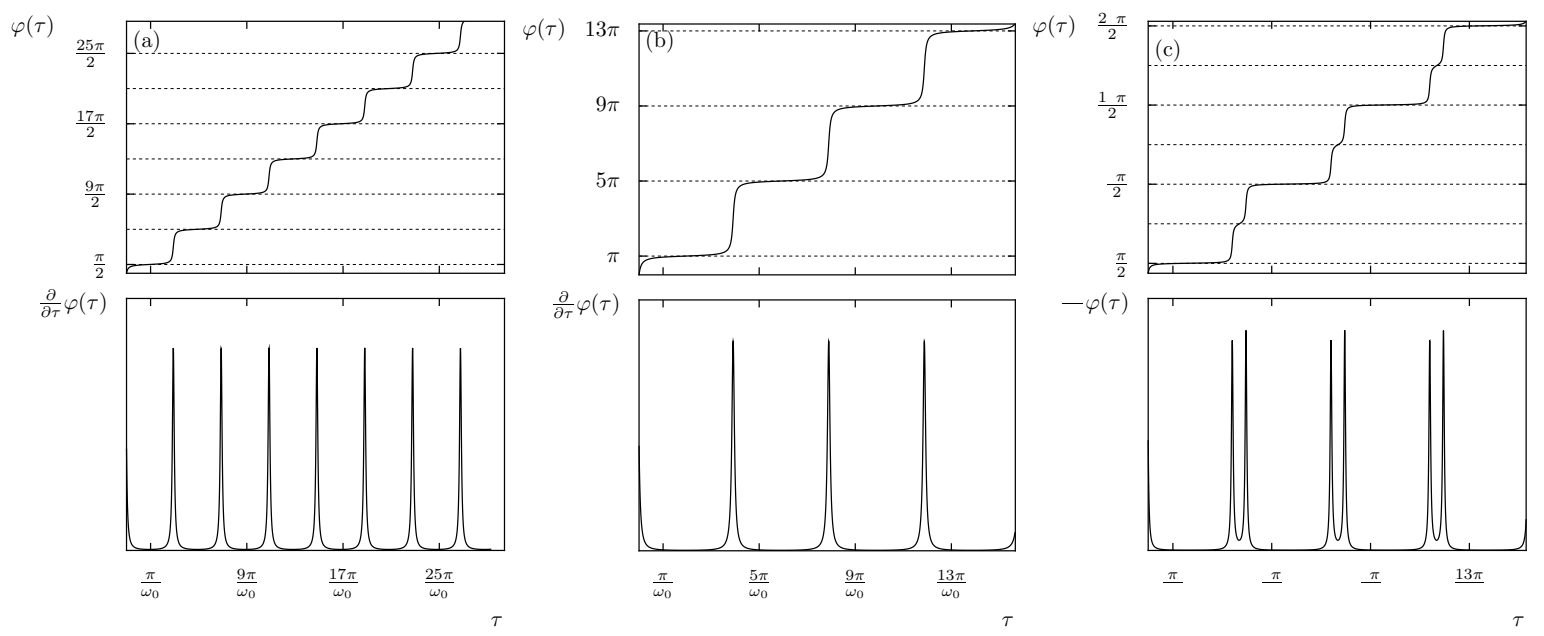

FIG. 10: $\varphi(\tau)$ (top row) and $\dot{\varphi}(\tau)$ (bottom row) as a function of time with a value of $\alpha_{0}$ close to the critical value $\alpha_{c}$ in a $2 \pi$, $4 \pi$ and mixed situation with $\alpha_{M}=1 / 15$, from left to right.

in units of $2 e / \hbar$. That is, the frequency of the junction is proportional to the induced voltage. In summary, by increasing $\alpha_{0}$ we modify the frequency of the junction $\omega_{0}$, and we also provoke an increment of the slope between stairs, which produces a widening of the peaks seen in $\dot{\varphi}(\tau)$.

Undriven system with the Majorana mode

We add now the Majorana mode by including the term $\alpha_{M} \sin (\varphi / 2)$ to the supercurrent yielding

$$
\dot{\varphi}(\tau)=\alpha_{0}-\sin (\varphi)-\alpha_{M} \sin (\varphi / 2)
$$

with $\alpha_{M} \ll 1$. In the example presented here we have used the value $\alpha_{M}=1 / 15$, so that the sum of this term modifies slightly $\sin (\varphi)$. However, as we can observe in panel c of Fig. 11. the periodicity of $\varphi(\tau)$ is drastically modified for values of $\alpha_{0}$ close to the new critical value, $\alpha_{c} \approx 1+\alpha_{M} / \sqrt{2}$ (see panel $\mathrm{c}$ of Fig. 10). The solution $\varphi(\tau)$ turns from a $2 \pi$ periodicity to a $4 \pi$ for values of $\alpha_{0}$ close to the critical value $\alpha_{c}$ (see Fig. 10k) and becomes $2 \pi$ for larger values of $\alpha_{0}$ (see Fig. 11 r). This effect is non-linear and makes a difference respect to the voltage biased experiment.

The change in the periodicity can be explained by means of analogous arguments as above. The addition of the Majorana mode may increase or decrease the duration of the flat regions depending on the $\operatorname{sign}$ of $\sin (\varphi / 2)>0$. The time difference between the short and long periods increases as long as we are closer to the critical value $\alpha_{c}$. Thus, the $2 \pi$ periodic function turns to a $4 \pi$ periodic one as $\alpha_{0} \approx \alpha_{c}$, still with the characteristic frequency $\omega_{0}$. A more visual comparison can be made just by looking the similarities with a pure $4 \pi$ Josephson junction in panel b of Figs. 10 and 11.

We can study continously the transition from $4 \pi$ to the $2 \pi$ character of the junction calculating from numerics the largest frequency $\omega_{M}$ of the junction, defined by $\omega_{M}=2 \pi / \Delta \tau$, where $\Delta \tau$ is the dimensionless time difference between the two closest maximums of $\dot{\varphi}(\tau)$. When $\omega_{M}$ approaches $\omega_{0}$, the junction turns to be $2 \pi$ periodic. The general behavior is shown in Fig. 12, where we have plotted the relation $\omega_{M} / \omega_{0}$ vs. $\omega_{0}$ for three different values of $\alpha_{M}=1 / 15,1 / 10$ and $1 / 5$. The curves show the tendency of the periodicity of the junction as we increase $\omega_{0}$. We can observe that there is always a range of values of $\alpha_{0}$, i.e. a range of $\omega_{0}$, close to $\alpha_{c}$ where the Majorana mode imposes its $4 \pi$ periodicity to the junction. Roughly speaking, this range is of the order of $\alpha_{M}$. The dashed curve placed at $\omega_{M} / \omega_{0}=1$ points out the tendency of the junction to behave $2 \pi$ periodically.

\section{Driven system with the Majorana mode}

Until now we have seen that the phase presents a range of voltages or frequencies where its dynamics is governed by the periodicity of the Majorana mode. The question now is that if we will be able to measure the periodicity of 

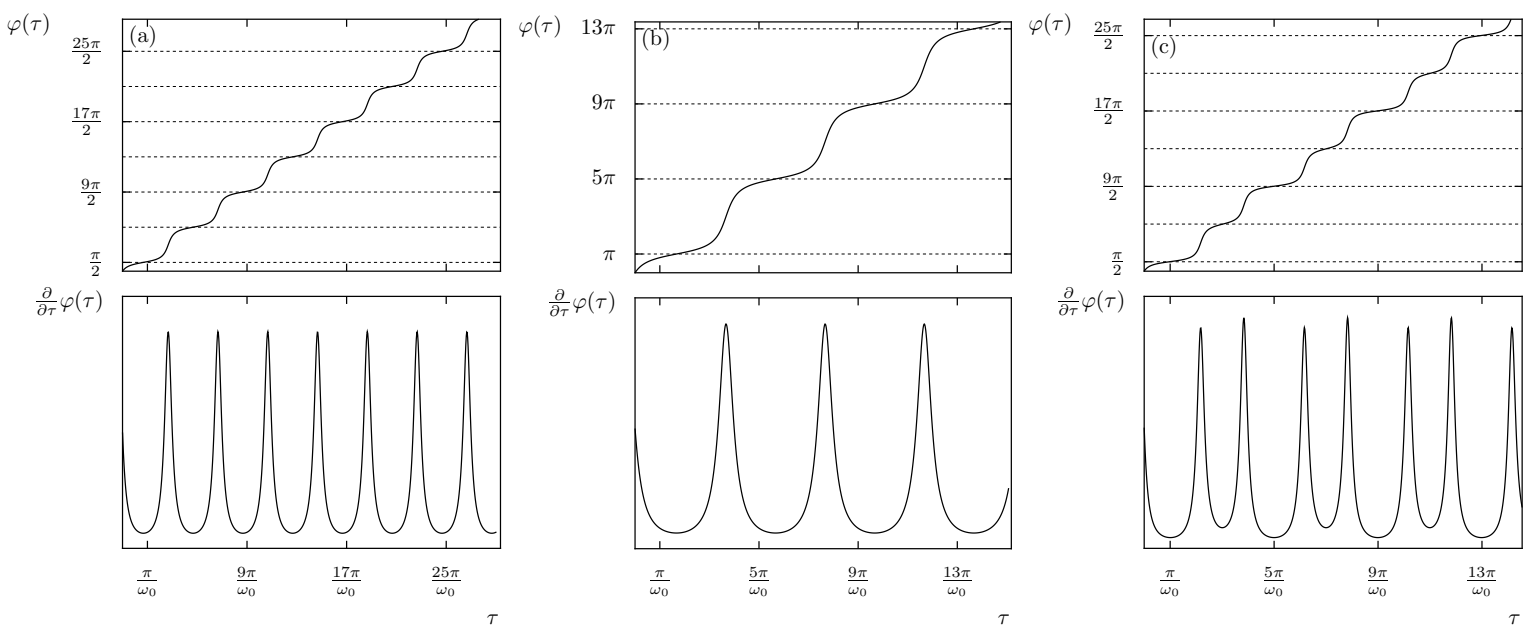

FIG. 11: Same plot as Fig. 10 for a larger value of $\alpha_{0}$ respect to the critical value $\alpha_{c}$. Comparing panel c of this figure and Fig. 10 we can appreciate the change of period when we move away from $\alpha_{c}$, i.e. when $\alpha_{0} \rightarrow \alpha_{c}$ (Fig. 11, $\rightarrow$ Fig. 10k) the periodicity of $\dot{\varphi}(\tau)$ approaches $4 \pi$

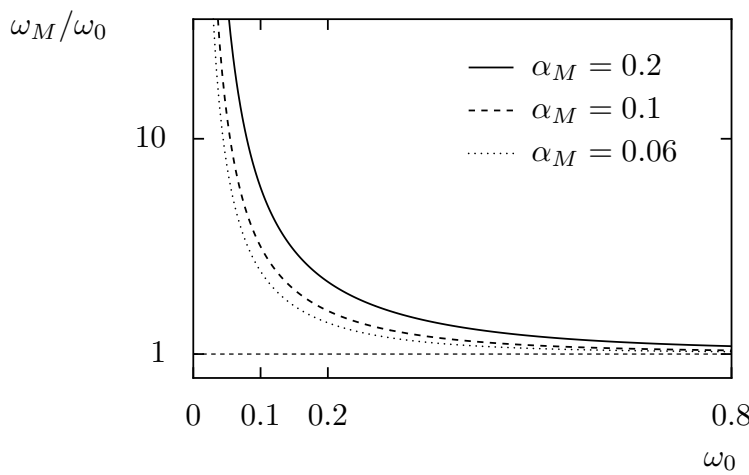

FIG. 12: Relation $\omega_{M} / \omega_{0}$ vs. $\omega_{0}$ for $\alpha_{M}=1 / 15,1 / 10$ and $1 / 5$ (from left to right). It represents the $4 \pi$-contribution of the junction as a function of the $2 \pi$, given by $\omega_{0}$. The range of values where $\omega_{M} / \omega_{0} \gg 1$ indicates the $4 \pi$ behavior of the junction. The dashed line corresponds to the value $\omega_{M} / \omega_{0}=1$, where is pure $2 \pi$ periodic.

the phase in that range of frequencies. Typical procedure to measure the periodicity implies to drive the phase by means of an ac current, that is

$$
\dot{\varphi}(\tau)=\alpha_{0}+\alpha_{1} \sin (\xi \tau)-\sin (\varphi(\tau))-\alpha_{M} \sin (\varphi / 2)
$$

It is well known that the solutions $\varphi(\tau)$ at the steps are phase-locked solutions (e.g see Ref. 8 ). This means that the driving force imposes its frequency to the driven system. And in this way, the solutions of $\dot{\varphi}(\tau)$ change from the former frequency imposed by $\alpha_{0}$, i.e. $\omega_{0}$, to the ac frequency $\xi$.

This special property of the phase locked solutions is very important because it allows to access the range of frequencies where the junction behaves $4 \pi$ periodically: Choosing $\alpha_{M} \gtrsim \xi$, that is, the order where we have seen that $\omega_{M} / \omega_{0} \gg 1$, will lead to have a dominant $4 \pi$ periodicity, which leads to an even Shapiro spectrum. We can see this behavior in the plots shown in Fig 13, where we have plotted the Shapiro steps for different values of $\alpha_{M}$. We see how the odd steps tend to vanish for $\alpha_{M} \approx \xi$. Finally, we have plotted in Fig. 14 the height of the first four steps as a function of the ac intensity $\alpha_{1}$ for $\alpha_{M}=0.15$ and $\xi=0.1$. We can observe a clear predominance of the even steps, for the whole range of $\alpha_{1}$. Remarkably, we see that for $\alpha_{1} \approx \alpha_{M}$, odd steps are zero. This behavior can be explained by the fact that in our reasoning we have implicitely considered that $\alpha_{1}$, reads out the periodicity of the junction at the imposed frequencies $\xi$. In other words, we have considered that the effect of adding the ac current is to select the frequency of the junction, without introducing its $2 \pi$ periodicity, and in such sense $\alpha_{1}$ needs to be of the order of 
$\alpha_{0}$

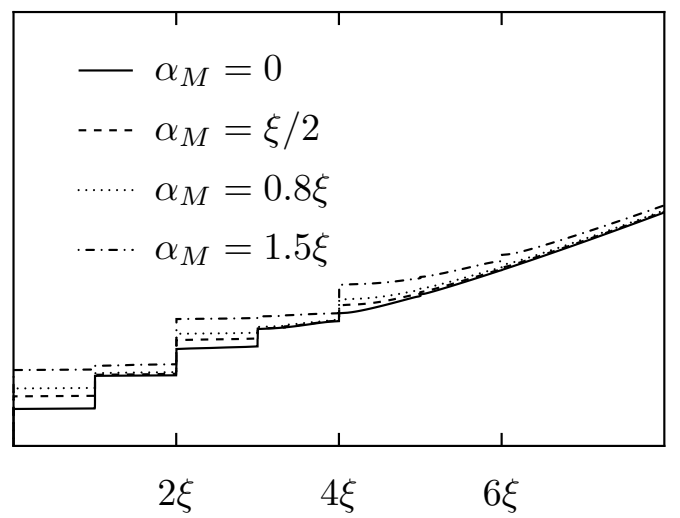

$\alpha_{0}$

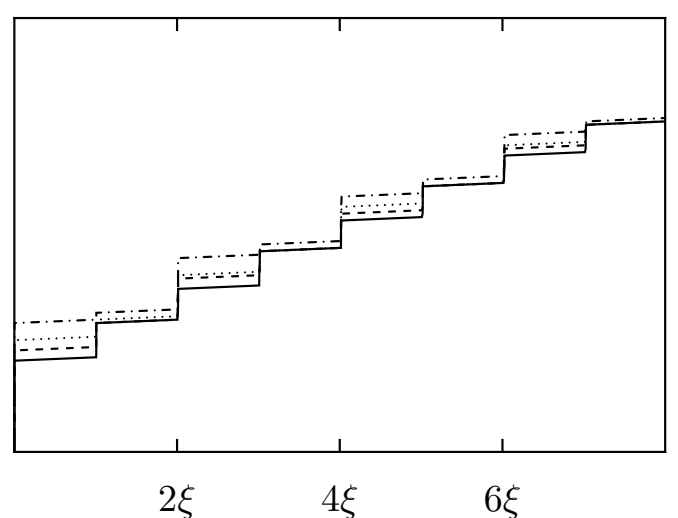

FIG. 13: Shapiro I-V curves for $\alpha_{1}=0.3$, and $\xi=1 / 5$ for the left plot and $\xi=1 / 20$ for the right plot. The value of $\alpha_{M}$ is increased from 0 to $1.5 \xi$ in both plots. We can appreciate the reduction of the odd steps as long as $\alpha_{M} \gtrsim \xi$.

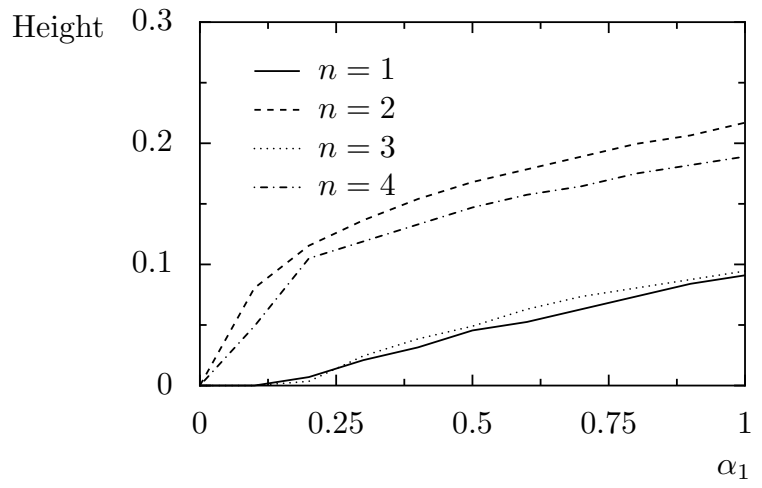

FIG. 14: Height of the first four steps as a function of $\alpha_{1}$ for $\alpha_{M}=0.15$ and $\xi=0.1$.

$\alpha_{M}$. We see that for larger values of $\alpha_{1}$, odd steps coming from a $2 \pi$ contribution become larger.

\section{Conclusions}

In this section we have explained numerically the behavior of the solutions $\varphi(\tau)$ and $\dot{\varphi}(\tau)$ of the current biased Shapiro experiment in the presence of a Majorana and several normal modes. We have given the explanation of the dominance (for a range of frequencies) of the even Shapiro steps in the presence of an, in principle, negligible $4 \pi$ contribution. In order to understand this behavior we have explored the solutions of the undriven system and seen that in general there is always a region of frequencies where the $4 \pi$ periodicity dominates. This region has an interval of frequencies of the order of $\omega_{0} \lesssim \alpha_{M}$, where $\alpha_{M}$ is the dimensionless intensity of the Majorana mode. Therefore, if one wants to measure some signature of the $4 \pi$ periodicity of the system, it will be needed that the measurement is performed in this frequency regime.

One of the advantages of the current biased Shapiro experiment consists on the fact that the steps present phase locked solutions. This means that the forced system imposes its periodicity to the junction. Therefore, we can impose the ac frequency $\xi$ to the junction $\omega_{0}$ by means of biasing the junction by an ac current. And meanwhile measure the periodicity of the junction by looking at the positions of the steps. We have seen that when $\alpha_{M} \gtrsim \xi$ even Shapiro steps dominate, and also that this behavior is more robust when $\alpha_{1} \approx \alpha_{M}$. Transforming back to physical units we have that taking into account that $I_{M}=1 \mathrm{nA}$ and $R=3 \mathrm{k} \Omega$, then $\omega_{a c} \lesssim 2 e R I_{M} / \hbar=10^{10} \mathrm{~Hz}$. 
[1] A. Y. Kitaev, Phys. Usp. 44, 131 (2001).

[2] M. Leijnse and K. Flensberg, Phys. Rev. B (R) 84, 140501 (2010).

[3] S. N. Shevchenko, S. Ashhabb, and F. Nori, Phys. Rep. 492, 1 (2010).

[4] H. J. Kwon, K. Sengupta, and V. M. Yakovenko, Eur. Phys. J. B 37, 349 (2003).

[5] R. M. Lutchyn, J. D. Sau, and S. D. Sarma, Phys. Rev. Lett. 105, 077001 (2010).

6] Y. Oreg, G. Refael, and F. von Oppen, Phys. Rev. Lett. 105, 177002 (2010).

[7] L. G. Aslamazov and A. I. Larkin, JETP 9, 87 (1969).

[8] E. D. Thompson, J. Appl. Phys. 44, 5587 (1973).

[9] M. Kvale, S. E. Hebboul, and J. C. Garland, Physica B 165, 1585 (1990). 